\title{
L'OUVERTURE
}

\section{DU NOUVEAU chenal D'aceis AU PORT DE ROUEN DANS L'ESTUAIRE DE LA SEINE}

PAR

\section{P. CHAPON *}

1. L'amélioration des conditions d'accès au port de Rouen a nécessité un important aménagement de l'estuaire de la Seine pour y stabiliser un chenal de navigation dont les profondeurs soient augmentées d'environ $3 \mathrm{~m}$ par rapport à celles des chenaux de l'estuaire libre et puissent être maintenues moyennant des dragages économiquement acceptables.

Les conditions naturelles de l'estuaire de la Seine sont très défavorables à l'établissement naturel d'un chenal stable et profond : le marnage est de $8 \mathrm{~m}$ en vive-eau; les courants ont une intensité atteignant 5 nœuds, et une action dissymétrique en flot et en jusant, mobilisant d'importantes quantités de sables fin (diamètre moyen : $1 / 10^{\circ}$ de mm) qui constituent le fond de la partie aval de la rivière et de son embouchure. L'estuaire a la forme d'un entonnoir fortement convergent vers l'amont, qui introduit une brusque discontinuité du calibrage de la rivière sur les trente derniers kilomètres de son parcours.

Comme il est de règle pour les rivières naturellement peu profondes, seule une importante modification de l'embouchure pouvait produire une appréciable augmentation des profondeurs offertes à la navigation. Sans prétendre bouleverser les conditions naturelles de l'écoulement, il était indispensable de supprimer certaines causes de l'instabilité et de la médiocrité du chenal : il fallajt pour cela concevoir un tracé de calibrage assurant un raccordement progressif entre la partie endiguee de la rivière en amont de Tancarville, et la baie de la Seine, et permettant d'organiser un circuit des matériaux solides avec formation de dépôts en

* Ingénieur des Ponts et Chaussées à la Direction du Port de Rouen. dehors de la zone où devait être établi le chenal de navigation.

2. Comme il est d'usage pour l'étude des problèmes de ce genre, le tracé fut étudié sur un modèle réduit à fond mobile. Après de longs et minutieux essais de tarage, le modèle a montré qu'il était possible de stabiliser un chenal dont les profondeurs soient effectivement accrues d'environ 3 mètres.

Ce chenal devait être appuyé contre une digue concave, prolongeant jusqu'à Honfleur le tracé de la berge en amont de la Risle. L'effet de guidage des courants en aval de Honfleur est assuré par une digue submersible de $8 \mathrm{~km}$ de longueur, appelée digue du Ratier. Le calibrage est complété par la digue basse nord qui rétrécit la section par rapport à celle de l'estuaire libre (fig. 1).

3. La réalisation de ce projet entraînait donc la complète modification de la topographie de l'estuaire non aménagé, et nécessitait le déplacement de plus de 50 millions de mètres cubes de sable, la démolition de $6 \mathrm{~km}$ de digue en enrochements et en béton, et la construction de plus de $30 \mathrm{~km}$ de nouvelles digues.

3.1. Deux méthodes pouvaient être envisagées pour réaliser la modification de tracé du chenal dans la partie centrale de l'estuaire, où le problème paraissait a priori le plus délicat:

a) La première consistait à draguer la digue sud existante entre le confluent de la Risle et Honfleur, puis le banc de sable qui s'était constitué au sud de cet ouvrage, et à déplacer ainsi progressivement le chenal vers son tracé définitif.

b) La seconde, au contraire, consistait à creuser le nouveau chenal suivant le tracé du projet sur la plus grande longueur possible, à l'abri d'ouvrages interdisant sa communication avec le reste de l'estuaire, puis à raccorder cette section du nou- 


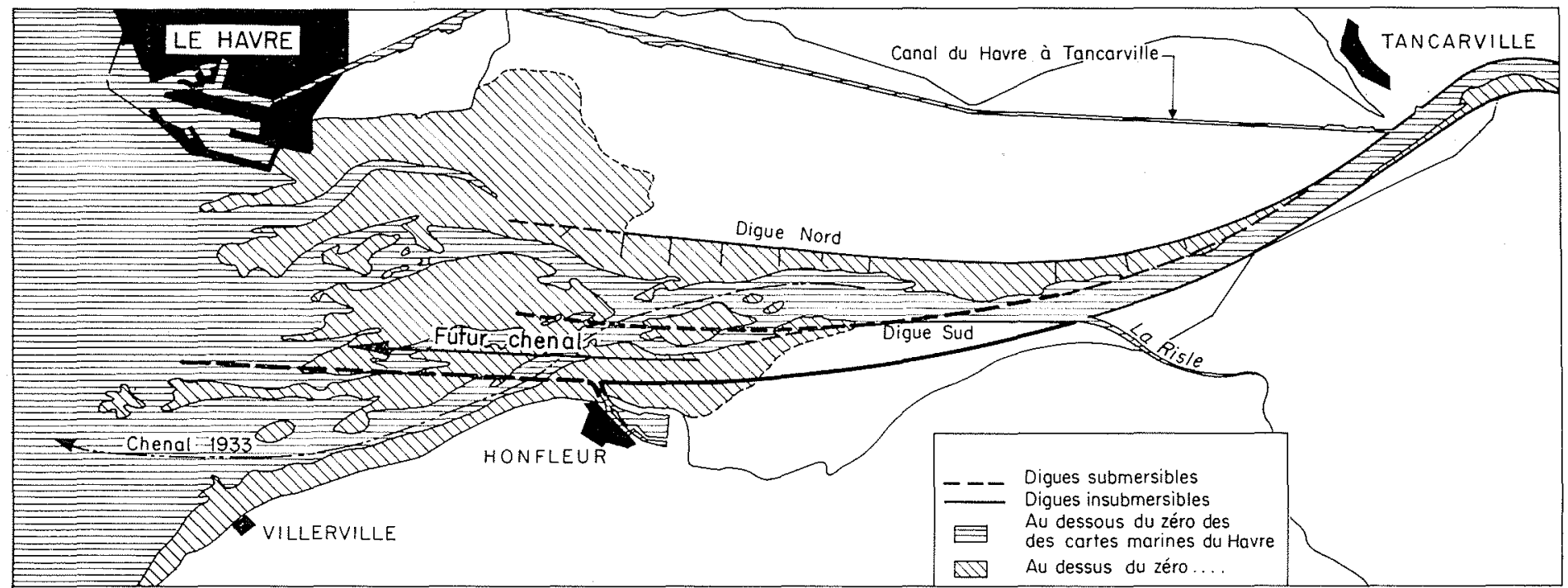

1/ Tracé du projet d'aménagement de l'estuaire de la Seine.

General outline of the Seine estuary development scheme.

veau chenal à la partie maintenue de l'ancien, en pratiquant une brusque coupure des ouvrages ou des bancs qui les séparaient.

Dans les deux cas, la méthode utilisée devait réduire au strict minimum la gêne apportée à la navigation (qu'il ne pouvait être question d'interrompre), et en particulier limiter la durée et l'importance des perturbations causées aux profondeurs des chenaux jusqu'à la stabilisation des fonds suivant le nouveau tracé.

3.2. La méthode progressive présentait l'avantage de contrôler plus facilement l'opération du changement de chenal, dont le déplacement vers son tracé définitif aurait été réalisé de façon quasi continue. Elle exigeait cependant la mise en œuvre d'un très important matériel de dragages pour éviter une durée excessive. Elle nécessitait la construction d'importants ouvrages nord -- par exemple sous forme d'épis - pour maintenir sensiblement constante la section mouillée et éviter que, pendant une longue période, l'écoulement s'effectuât dans une section trop large qui aurait entrainé la formation d'atterrissements gênants pour la navigation; ces ouvrages nord n'auraient par la suite présenté qu'un faible intérêt pour le calibrage définitif du chenal.

3.3. La méthode brutale était évidemment plus séduisante, car on pouvait penser qu'elle réduirait la durée de la phase transitoire, pendant laquelle les profondeurs offertes à la navigation risquaient de se détériorer. Elle permettait, en outre, davantage que la précédente, d'utiliser les forces naturelles, et en particulier l'érosion des bancs de sable par les courants des grandes marées. Elle était, par contre, plus difficilement contrôlable, et les dépôts solides, inévitablement provoqués par la brutale augmentation de la section mouillée de l'écoulement, risquaient de se former dans la zone mème où devait être établi le futur chenal. On pouvait alors craindre que les moyens de dragages disponibles soient insuffisants à corriger cette évolution, et la plus élémentaire prudence exigeait de vérifier soigneusement sur le modèle réduit que la tendance naturelle de l'écoulement le conduisait vers le futur chenal (1).

3.4. En fait, l'ouverture du nouveau chenal dans l'estuaire de la Seine a procédé à la fois de l'une et l'autre méthodes et de leur combinaison.

En aval de Honfleur, où l'estuaire était très large et les courants plus faibles, c'est la méthode progressive qui a été utilisée pour donner au chenal son tracé définitif. Au contraire, en amont de Honfleur où existait un certain calibrage donnant des courants dont l'intensité dépassait 5 nœuds en viveeau, le chenal a été ouvert de façon brutale, mais son calibrage définitif a été plus lent, et l'augmentation du débit dévié dans le nouveau tracé a été provoquée par la fermeture progressive de l'ancien chenal.

4. Au moment où furent entrepris les travaux préparatoires à l'ouverture du nouveau chenal, c'est-à-dire vers 1957 , la navigation empruntait un chenal qui longeait la côte du Calvados en aval de Honfleur et débouchait en baie de Seine au sud du banc du Ratier; des dragages d'entretien effectués en permanence à son inflexion, vers le méridien de Honfleur, avaient permis de donner à ce chenal un relative stabilité depuis 1945 ; le centre de l'estuaire était alors occupé par un important banc «en flèche», creusé à sa partie extérieure et centrale par une grande fosse de flot.

Le modèle avait montré que la digue du Ratier assurait dès sa construction une concentration des courants sur sa face nord, suffisante pour entailler le banc et ouvrir le nouveau chenal. En fait, les

(1) Encore convenait-il de n'accepter les conclusions du modèle, sur ce point particulier, qu'avec une certaine réserve, car les phénomènes d'érosion de bancs découvrants, ou insubmersibles, par les courants de maré, ne sont pas tout a fait comparables à l'action des courants sur le modelé des fonds sous-marins.

(2) Les ouvrages sont repérés en plan par rapport au quadrillage kilométrique NS/EW dont le zéro est situé au phare sud de la Hève. 
circonstances naturelles se montrèrent particulièrement favorables à l'opération.

La construction de la digue du Ratier a été entreprise dès 1957 à partir du méridien 9 (2) (3) vers l'aval, puis, dès le début de l'année 1958 , à partir de son extrémité aval au méridien en remontant vers l'amont.

Mais, en même temps que progressait la construction de la digue du Ratier, on constatait que la fosse creusée par le flot à la face extérieure du banc s'accentuait et que le chenal avait tendance à la rejoindre, tandis que l'inflexion du chenal de navigation se dégradait au droit de Honfleur: le chenal tendait donc à suivre le tracé central qu'il avait en 1939 (4).

L'action des dragues aspiratrices en marche a permis de hâter cette évolution, et l'ancien chenal a pu être abandonné par la navigation dans le courant de l'année 1958. A cette date, le chenal suivait done sensiblement le tracé du projet en aval du méridien 8, mais, en amont de cette limite, il était séparé de la côte du Calvados par un banc de sable dont les cotes dépassaient le niveau $(+1,00 \mathrm{~m})$ audessus du zéro des cartes marines du Havre; d'importants dragages étaient donc nécessaires pour ouvrir le nouveau chenal vers l'amont, à travers ce banc.

5. On ne pouvait évidemment pas compter sur la seule action naturelle de la marée pour déplacer le chenal en amont de Honfleur, mais on a cherché à utiliser au maximum les forces naturelles, en même temps qu'à réduire au minimum la durée de la période transitoire pendant laquelle s'effectuerait la stabilisation du chenal suivant son nouveau tracé (fig. 2).

5.1. Le chenal a été dragué sur environ $7 \mathrm{~km}$ de longueur, approximativement à sa cote et sa largeur définitives dans un bassin creusé an sud de la digue existante, et limité au sud par la nouvelle berge qui pouvait ainsi être construite dans d'excellentes conditions techniques. Ce bassin était fermé à son extrémité ouest, sur le banc de Saint-Sauveur, par un digueron en sable insubmersible; la communication entre l'estuaire et le plan d'eau intérieur était assurée par une porte levante qui donnait le passage des engins de travaux et commandait le niveau du bassin; ce procédé permettait d'exécuter les dragages à l'abri de la houle et des courants, dans un plan d'eau à niveau sensiblement constant (fig. 3).

5.2. Le principe de l'ouverture du bassin consistait à amorcer le chenal dans le banc de SaintSauveur par le dragage d'un sillon d'une cinquantaine de mètres de largeur, et à provoquer un écoulement brutal du bassin dans ce sillon, en pratiquant une brèche dans le digueron en sable au moment opportun. Le modèle réduit avait montré que les violents courants dus à la vidange et au remplissage du bassin permettaient un élargissement très rapide du lit du futur chenal à travers le banc de Saint-Sauveur.

(3) Il était évidemment impossible d'entreprendre la construction de la digue depuis la côte du Calvados, car il était nécessaire de maintenir le chenal de navigation dans cette zone tant qu'il débouchait au sud du banc du Ratier.

(4) Il avait suivi ce tracé central pendant toutes les périodes «actives» de la marée, qui comportaient de fréquentes marées de forts coefficients $(1905,1921,1939, \ldots)$ mais ce tracé apparaissait comme naturellement instable.
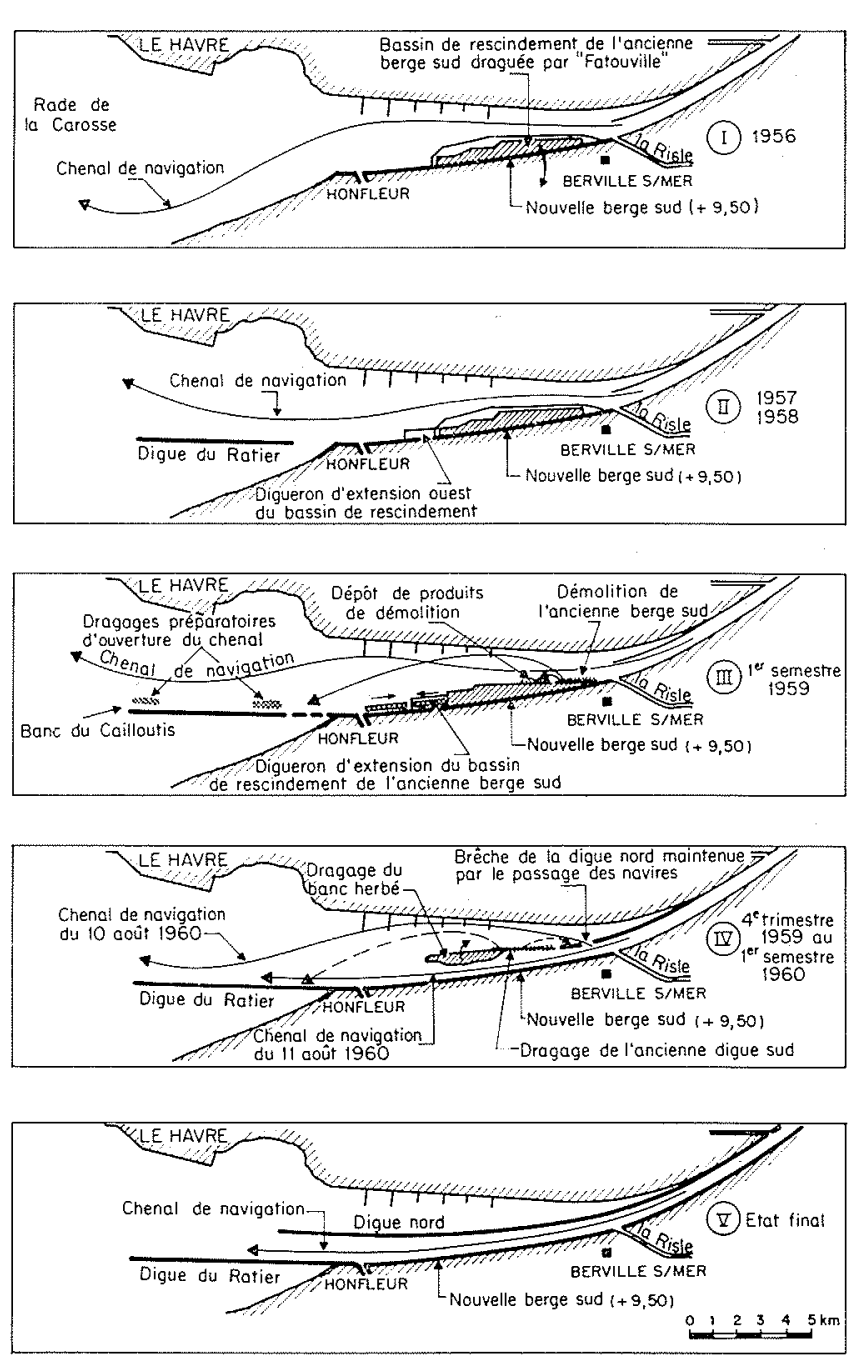

2) Schéma des opérations de changement du chenal de navigation.

Shipping channel diversion operations.

Il ne pouvait en être de même à l'amont, où le bassin n'était pas fermé par un ouvrage et un banc en matériaux mobiles, faciles à entrainer par de forts courants. L'enlèvement instantané de la digue bétonnée du modèle réduit et son ripage à l'emplacement de la future digue nord ne prétendaient évidemment pas représenter la réalité. Par ailleurs, le dragage du bassin lui-même, qui nécessitait l'enlèvement de 40 millions de mètres cubes de sable, devait durer plusieurs années, et de grandes précautions devaient être prises pour éviter une ouverture prématurée qui aurait inévitablement entraîné le comblement de la partie draguée. L'ouverture du bassin par l'amont devait donc être amorcée de façon brutale, car il était impossible de le laisser pendant longtemps ouvert seulement par l'aval, sous peine de le voir rapidement remblayé, mais par ailleurs, la complète ouverture amont ne pouvait être réalisée sans intervention d'un puissant matériel de dragage. En fait, l'opération devait être conduite de façon à accélérer le dragage de la digue, sans pour autant affaiblir trop hâtivement la digue et courir le risque d'une ouverture prématurée du bassin.

5.3. Afin de réduire l'importance de l'érosion naturelle du banc de Saint-Sauveur, demandée à 


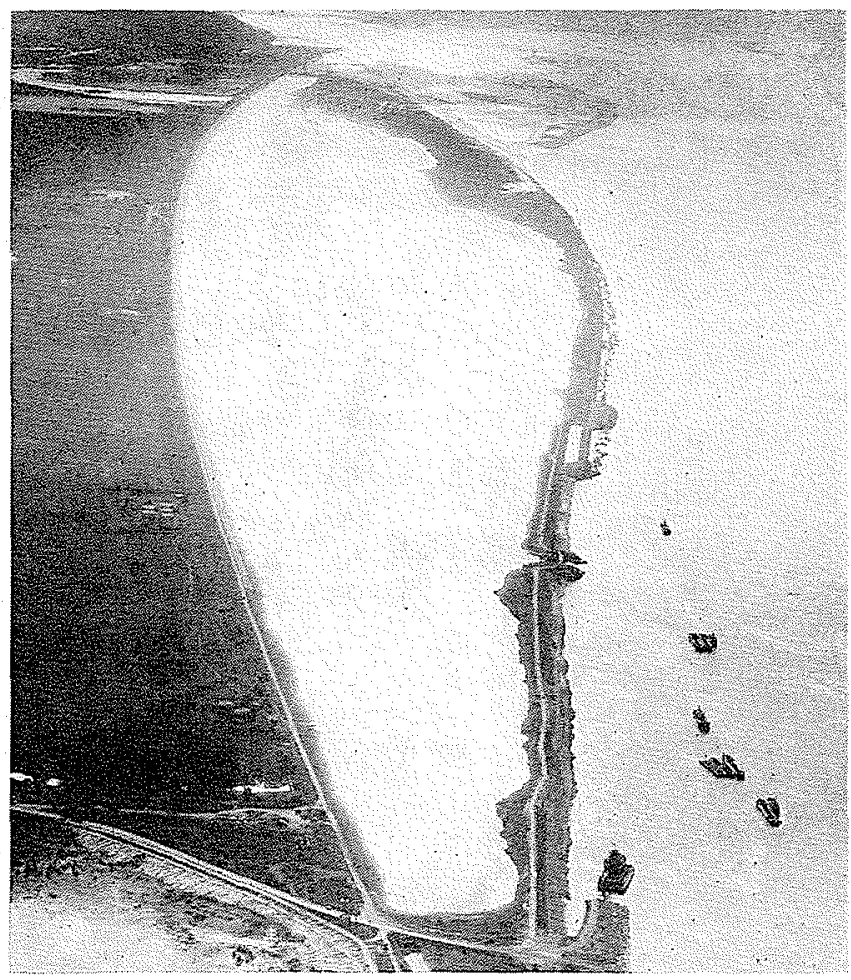

3/ Photo aérienne. Le bassin avant son ouverture (Ia photographie est prise depuis la partie amont de l'estuaire, le haut de la photographie correspondant a la direction onest):

à droite, l'ancienne digue sud, à gauche la nouvelle berge).

An aerial view of the basin before it was opened up, locking from the river end of the estuary towards the west at the top of the picture.

The old south dylie is on the right

and the new embankment can be seen on the left.

l'action des courants de remplissage et de vidage du bassin au moment de son ouverture, la zone draguée en bassin fermé a été poussée aussi loin que possible vers l'ouest.

Au cours de l'été 1959, un sillon a été creusé sur $50 \mathrm{~m}$ de largeur par une drague aspiratrice-refouleuse stationnaire, à partir de Honfleur vers l'amont, parallèlement à la nouvelle berge sud, jusqu'à une centaine de mètres du digueron -- distance jugée suffisante pour éviter tout risque de glissement.

Le 28 septembre 1959, au début de la grande vive-eau d'équinoxe, après la fin de l'étale de la pleine-mer du matin, une brèche fut pratiquée dans le digueron au moyen d'une dragline; l'eau du bassin, qui avait été rempli au maximum et qui atteignait le digueron par un petit sillon de quelques mètres de largeur creusé depuis la digue au moyen d'une dragline, s'écoula par cette breche en direction du sillon extérieur; la baisse de niveau du plan d'eau extérieur augmentait rapidement et la différence de niveau avec le bassin accélérait l'écoulement qui érodait rapidement à la fois le digueron et le banc lui-même. Au moment de la basse mer, le courant avait une vitesse de $5 \mathrm{~m} / \mathrm{s}$ et le banc était creusé sur une quarantaine de mètres de largeur, à une cote comprise entre $(0,00)$ et $(+1,00)$. Plus de 15 millions de mètres cubes d'eau s'étaient écoulés par la brèche, et au moment où s'établit le flot le niveau du bassin était à peine plus élevé que celui de l'estuaire (fig. 4).

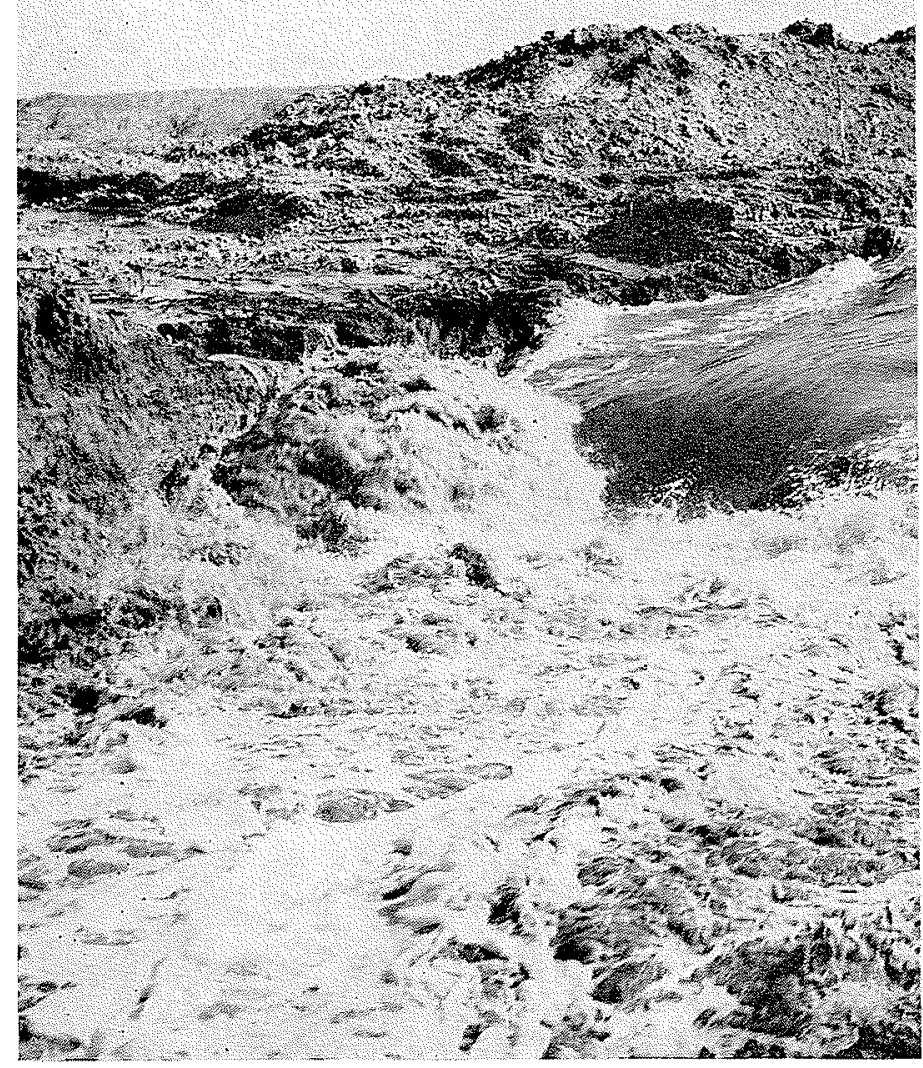

4/ Louverture aval du bassin, quelques dizaines de minutes a fût crevé.

The downstream basin opening shortly after the breaching of

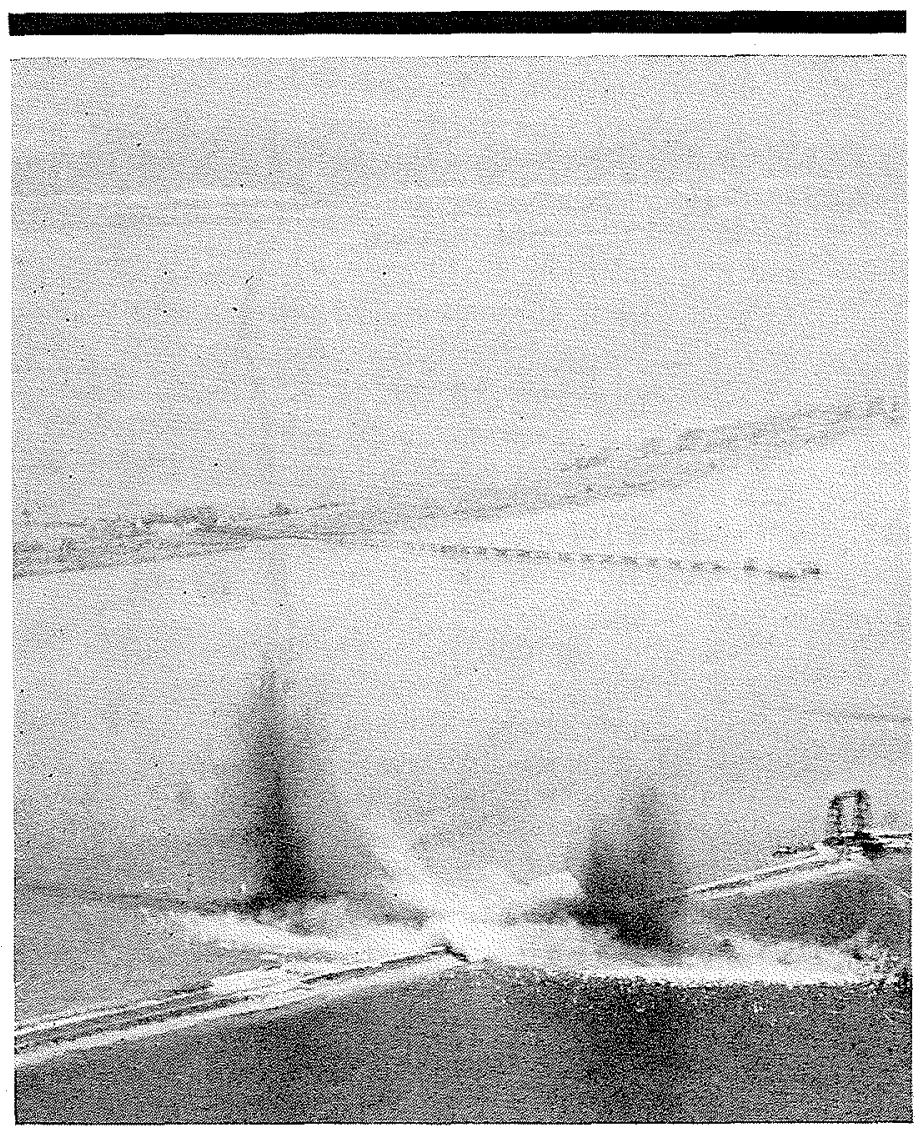

5/ L'ouverture amont, par sautage de deux charges d'explosifs, pour créer deux brèches dans l'ancienne digue sud. Blasting in progress at two points to open two breaches for the upstream opening. 


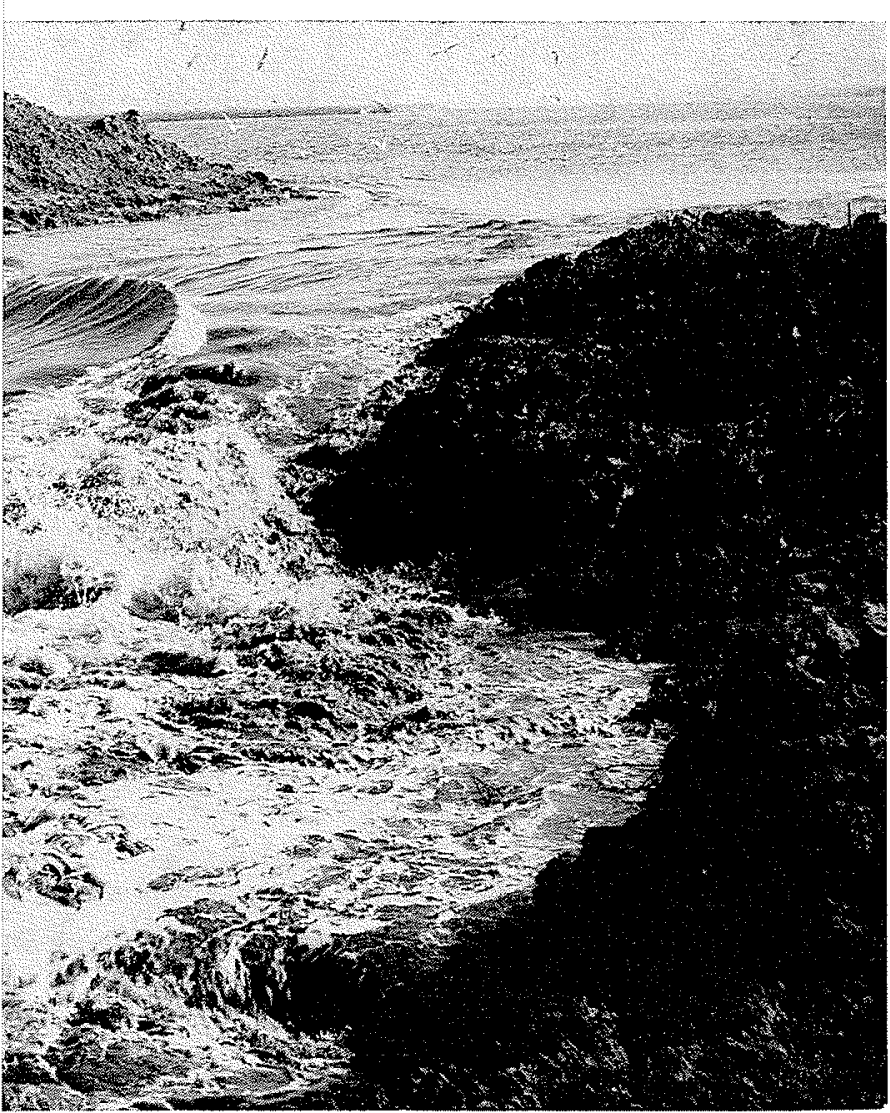

[ue Ie digueron

dyke.
En raison de la perte de charge due à l'étroitesse de la section de l'ouverture aval, la montée de niveau dans le bassin ne suivait pas la rapide variation du montant de l'estuaire, et la dénivellation correspondante qui dépassait $1,50 \mathrm{~m}$ après la mi-marée, produisait dans l'ouverture un courant de $4 \dot{a} 5 \mathrm{~m} / \mathrm{s}$ (5), qui érodait très rapidement le banc. Au bout de quatre marées, la largeur de l'ouverture atteignait $80 \mathrm{~m}$ sur la presque totalité de sa longueur et le déphasage entre le bassin et l'estuaire était réduit à quelques décimètres.

5.4. On a done décidé d'ouvrir le bassin par l'amont le 30 septembre, lors de la marée de plus fort coefficient de la période de vive-eau. Pour hâter cette ouverture et faire travailler au maximum les forces naturelles, on avait dragué les enrochements et le béton de la digue à démolir sur environ $2000 \mathrm{~m}$, mais on avait construit, en arrière de l'ancien perré, une contre-digue en sable, protégée dans sa partie supérieure par un revêtement en béton bitumineux de $15 \mathrm{~cm}$ d'épaisseur, et au pied par un talus de galets. Le revètement en béton bitumineux a été démoli au cours des journées qui ont précédé l'ouverture, afin de ne laisser subsister à ce moment que le corps de la digue en sable. Deux brèches d'une trentaine de mètres ont été pratiquées dans la digue le 30 septembre, au début du baissant, par l'explosion de deux fortes charges d'explosif (fig. 5).

La différence des niveaux entre le bassin et l'estuaire, qui subsistait malgré l'élargissement de l'ouverture aval était suffisante pour créer dans les

(5) La vitesse de remplissare du bassin était supéreure à celle du vidage en raison de la rapidité de la montée de la marée de lestuaire, qui jouait le rôle de «réservoir de mise en charge».

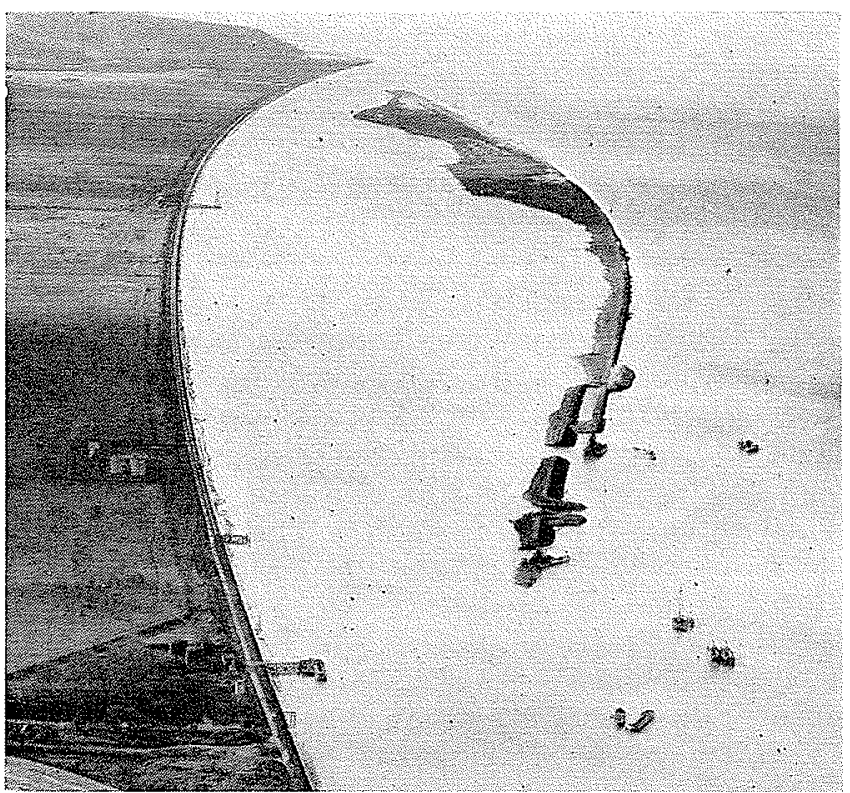

6/ Photographie aérienne de l'ex-bassin

une comparaison avec la figure 3 montre l'importance de l'ouverture aval

et fait apparaitre l'état d'avancement du dragage de l'ancienne digue sud.

An aerial view of the basin after it was opened, showing the wide downstream opening and dredging progress on the old south dylie. 
brèches des courants de près de $3 \mathrm{~m} / \mathrm{s}$. Au bout de quelques marées, ces courants avaient doublé la largeur des brèches et les avaient approfondies jusqu'au niveau des basses mers.

6. Les mouvements du bassin s'effectuaient donc par ses deux extrémités et le déphasage de son niveau par rapport à celui de l'estuaire était réduit pendant les plus fortes marées, mais les courants du remplissage par l'aval restaient cependant très violents et entrainaient d'importantes quantités de sable qui se déposaient dans la partie la plus large du bassin (fig. 6).

6.1. Afin de hâter l'érosion du banc de SaintSauveur et le calibrage du nouveau chenal, la drague «Fatouville» attaqua l'ouverture aval par passes successives parallèles à la nouvelle berge sud, d'une cinquantaine de mètres de largeur, en allant de l'amont vers l'aval, de façon à rester autant que possible abritée du fort courant de flot. Les matériaux dragués étaient refoulés au nord de l'ancienne digue sud, en bordure du chenal emprunté par la navigation. Près de 5 millions de mètres cubes de sable furent ainsi enlevés par la drague, tandis que l'érosion naturelle du banc et du fond à l'emplacement du nouveau chenal emportait une quantité au moins aussi importante de matériaux, à la fois dans le bassin et sur une barre extérieure en aval de l'entrée du port de Honfleur.

En même temps, une drague aspiratrice en marche perçait le bane qui existait au nord de la digue du Ratier entre les méridiens 8 et 10 , et son action était renforcée par l'effet de la digue qu'on prolongeait pour la raccorder à la côte du Calvados en aval de Honfleur. Dès le mois de février 1960, un chenal à la cote (0) avait pu être ouvert dans le banc.

6.2. Le dragage de l'ancienne digue sud a été entrepris à partir de chaque brèche, au moyen de deux draģues à godets. Les matériaux sableux étaient évacués en bordure de l'ancien chenal; les matériaux crayeux et les débris de béton, étaient, au contraire, déversés suivant le tracé de la nouvelle digue nord, de facon à en constituer l'infrastructure. Au mois de dcembre 1959 , le corps de l'ancienne digue était complètement dragué sur un kilomètre de longueur, mais la section transversale offerte à l'écoulement dans le nouveau chenal n'avait guère plus de $300 \mathrm{~m}$ de largeur. En avril 1960, alors que plus de $2 \mathrm{~km}$ de digue étaient dragués, la largeur du nouveau chenal n'atteignait pas $500 \mathrm{~m}$; encore faut-il souligner que la partie nord du bassin était encombrée d'un très important banc de sable et de vase qui s'était rapidement formé après son ouverture (fig. 7 et 8 ).

6.3. La majeure partie du débit empruntait encore l'ancien chenal, aussi bien en flot qu'en jusant; le nouveau chenal était en effet peu profond, en raison des apports qui s'étaient formés dans le bassin, alors que l'ancien chenal comportait, a la bifurcation amont entre les deux tracés, un mouillage profond provoqué par l'action de la nouvelle digue sud sur le jusant.

Cependant, en raison de l'étroitesse des sections, le courant gardait toujours une intensité assez forte (supérieure à 5 nouds dans le nouveau chenal) et, dès le mois d'avril 1960, l'érosion des dépôts qui s'étaient formés dans les parties centrales et amont du bassin était assez rapide; un chenal d'une cen- taine de mètres, à la cote $(0)$, était ouvert entre le confluent de la Risle et Honfleur au début de l'été 1960 .

6.4. Le détournement progressif, vers le nouveau chenal, du débit de jusant - et à un degré moindre du débit de flot - était favorisé par la construction de la digue nord qui avait été entreprise dès l'ouverture du bassin, à la fois par l'amont dans le prolongement de l'ouvrage existant, et par l'aval depuis l'ancienne digue sud. Au mois d'avril 1960, le corps de la digue nord était construit sur plus de $1500 \mathrm{~m}$ de longueur, en aval de l'ouvrage existant; en juin un tronçon d'un kilomètre était pratiquement achevé depuis l'ancienne digue sud, délimitant ainsi une passe de l'ordre de $300 \mathrm{~m}$ laissant le passage à la navigation.

Cette réduction de la section offerte à l'écoulement dans l'ancien chenal avait pour conséquence un important affouillement $d u$ fond en aval de l'ouvrage, traduisant une sérieuse augmentation locale de l'intensité du jusant (6) mais, en fait, le débit du jusant qui empruntait le nouveau chenal augmentait rapidement et les dépôts qui s'étaient constitués dans le bassin étaient entrainés vers l'aval où ils formaient des barres, en aval de Honfleur et même vers le méridien 8 .

Le flot restait très importait dans l'ancien chenal, au droit du méridien de Honfleur, et entraînait dans la partie nord de l'estuaire les matériaux arrachés aux barres et aux bancs extérieurs, mais sa force diminuait vers l'amont et les matériaux se déposaient par grandes quantités, engraissant l'extrémité est du banc de Saint-Sauveur, puis gagnant la totalité de la partie comprise entre les anciennes digues.

L'étroitesse des sections du nouveau chenal dans lequel était concentrée une partie croissante du débit de jusant augmentait les vitesses et provoquait la formation de fosses très profondes (plus de 8 mètres sous la plus basse mer locale) qui avaient certainement un effet très défavorable sur l'élargissement des sections et retardaient le calibrage; les matériaux arrachés par ces surcreusements allaient, en outre, alimenter les barres extérieures et constituaient des hauts fonds gènants pour la navigation.

7. Toutefois ce déblaiement du nouveau chenal en amont de Honfleur et l'accélération du remblaiement de l'ancien chenal ont conduit à décider l'ouverture du nouveau chenal à la navigation au cours de l'été 1960 : ouvert à titre d'essai aux petites unités le 11 juillet 1960 , le nouveau chenal fut effectivement mis en service au mois d'août (fig. 9).

Il importait, à partir de ce moment, de fermer au plus vite la «brèche» de la digue nord, pour concentrer le maximum du débit de jusant dans le nouveau chenal. La jonction des deux troncons de la digue nord nécessitait évidemment la mise en ouvre de moyens importants : une première campagne de déversements d'enrochements avait été effectuée par des chalands à clapets, à peu près régulièrement sur toute la longueur de la «brèche » de facon à constituer un seuil aussi hori-

(6) Afin de limiter l'érosion du fond à l'emplacement de la future digue, quelques déversements de galets ont été effectués, et ont effectivement assuré une certaine protection du fond, limitant ainsi la hauteur du tronçon de digue à construile. 


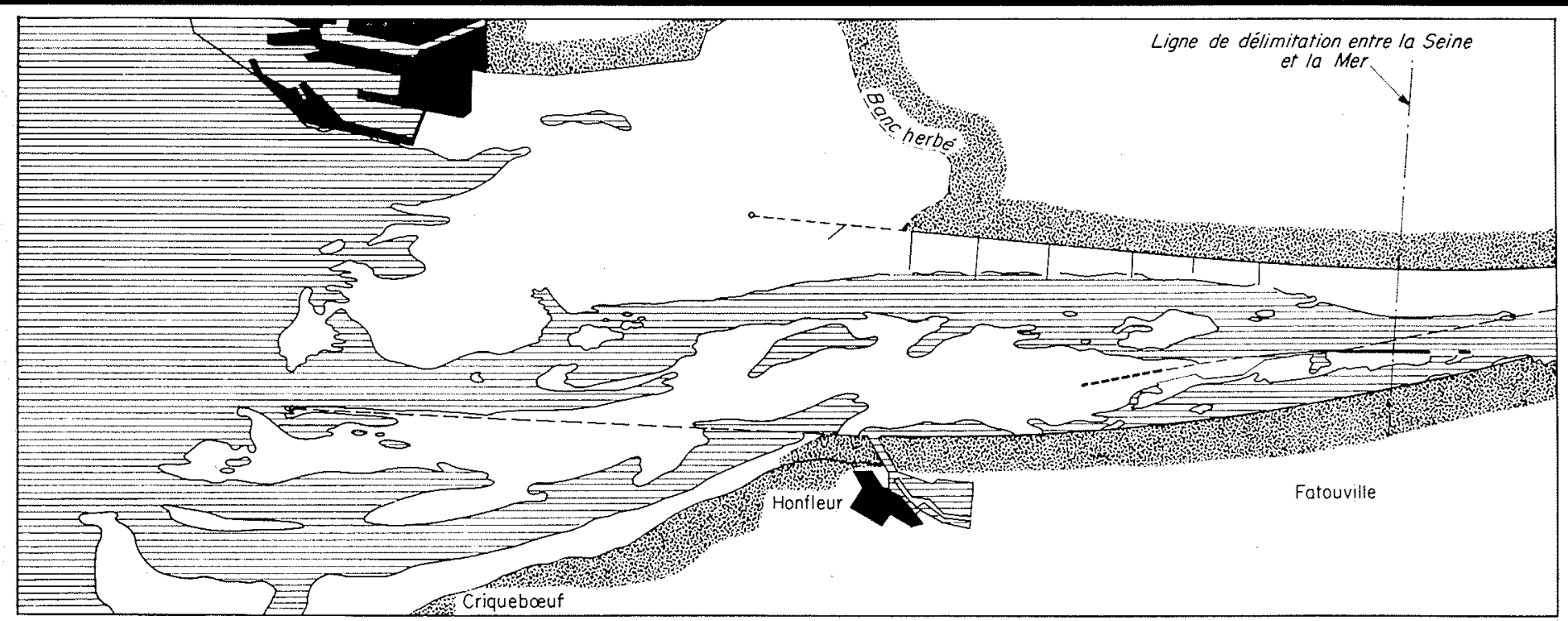

7/ Etat des fonds de l'estuaire au 31-10-59.

The estuary bed on October 31st, 1959.

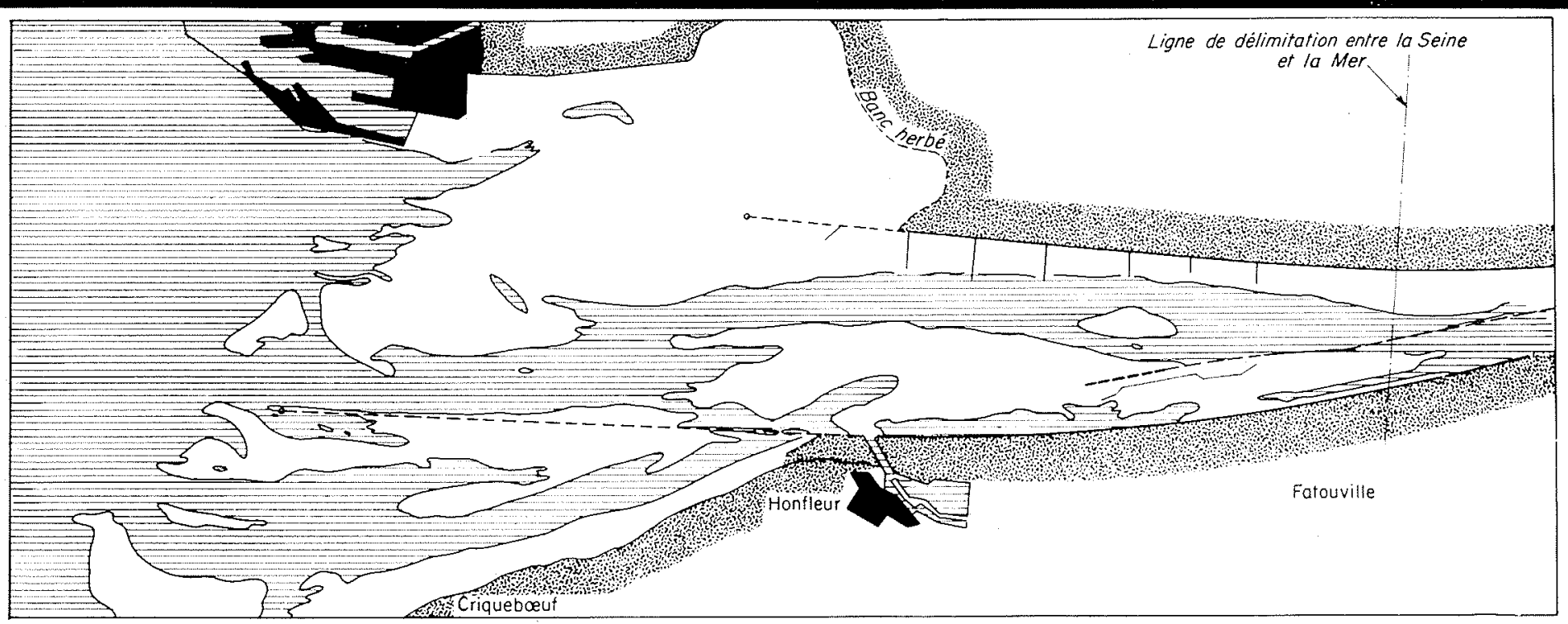

8) Etat des fonds de l'estuaire au 31-3-60.

The estuary bed on March 31st, 1960.

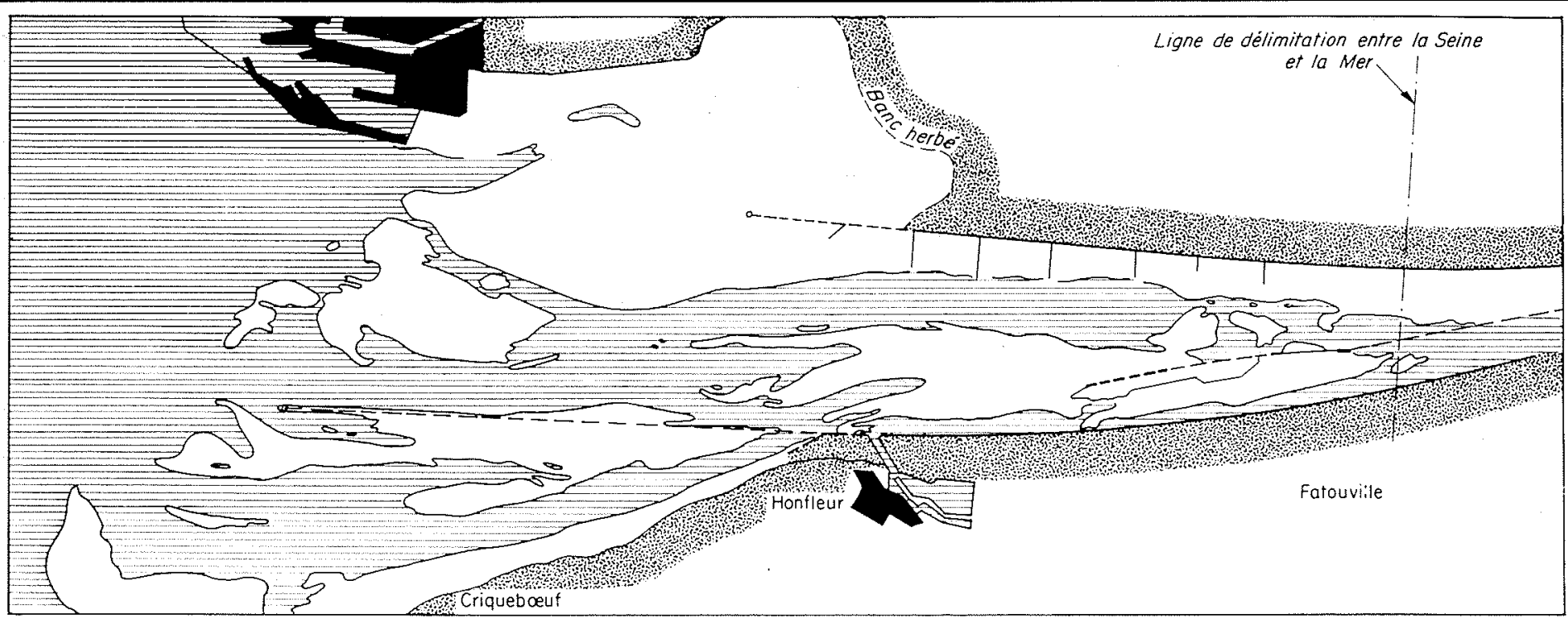

9/ Etat des fonds de l'estuaire au 30-6-60.

The estuary bed on June 30th, 1960. 


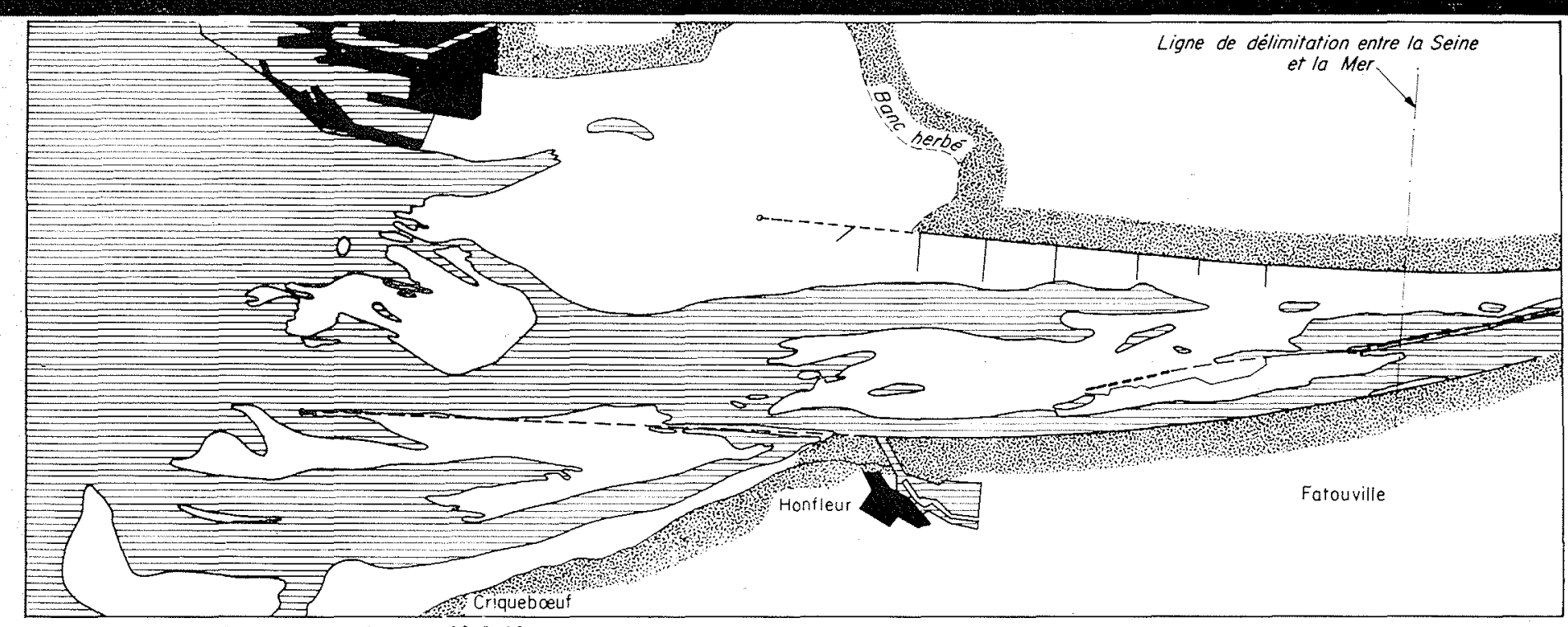

10/ Etat des fonds de l'estuaire au 30-9-60,

The estuary bed on September $30 t h, 1960$.

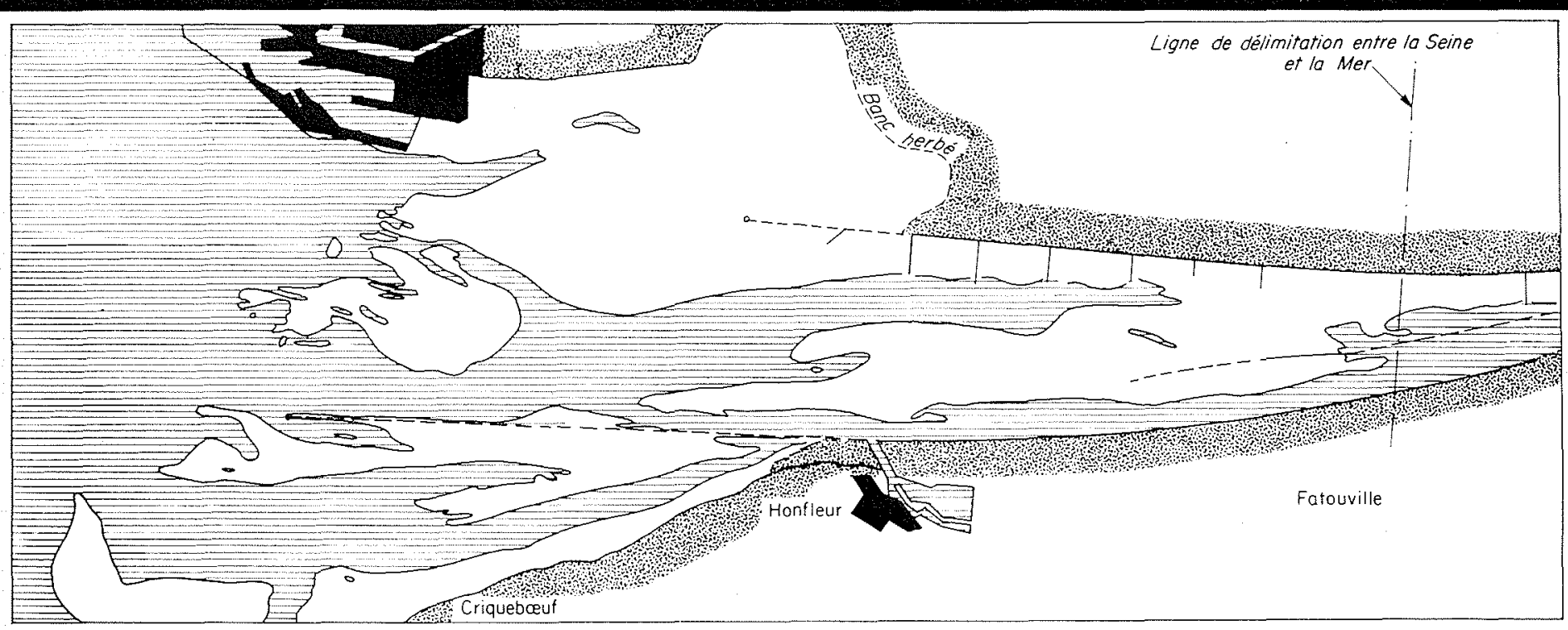

11/ Etat des fonds de l'estuaire au 31-12-60.

The estuary bed on December 31st, 1960.

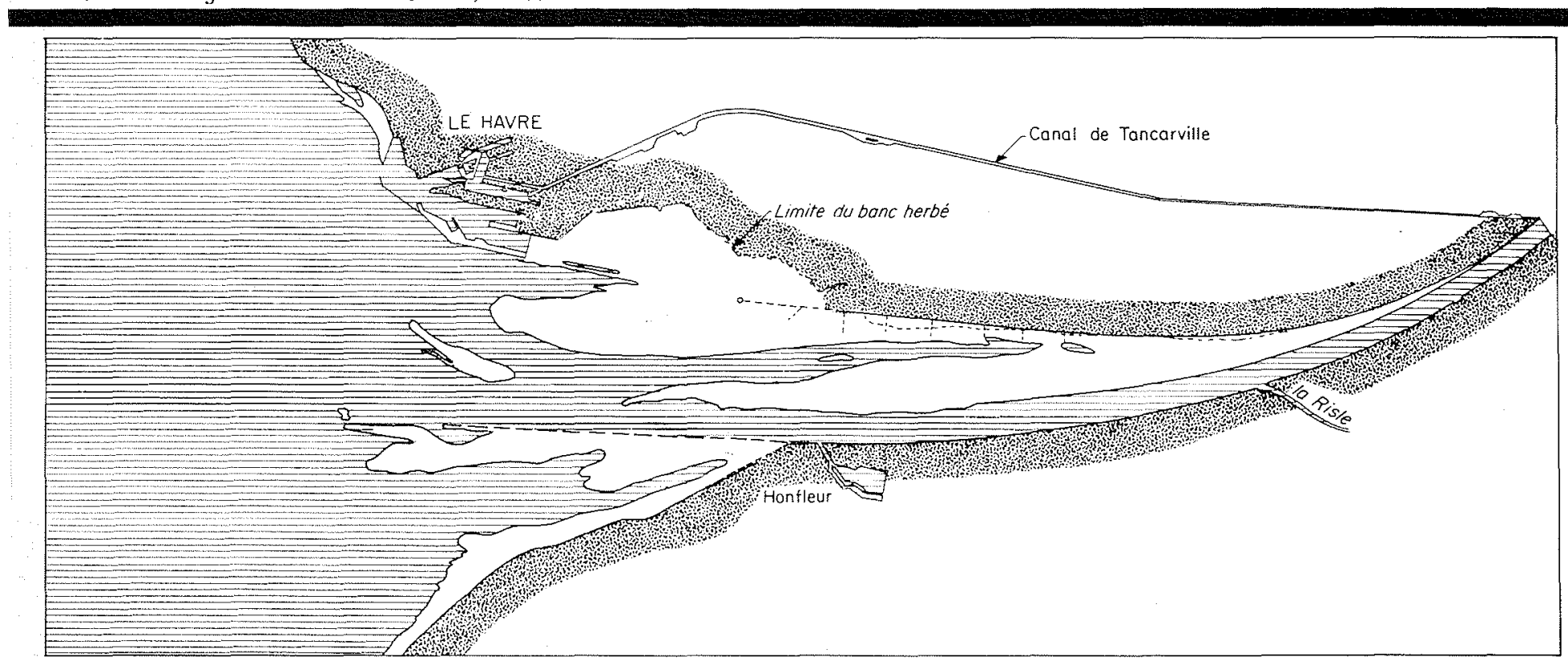

12/ Etat des fonds de l'estuaire au 29-2-64.

The estuary bed on February $29 t h, 1964$. 
zontal que possible, puis de nombreux enrochements furent déversés, également par couches horizontales, par un élévateur à déblais qui était alimenté par des chalands de dragages à une cadence de 3000 à $4000 \mathrm{~m}^{3}$ par jour (fig. 10).

A la fin du mois de septembre, la digue nord était arasée à la cote $(+3,50)$, et pratiquement, dès que la marée baissait au-dessous de la cote $(+4,00)$, la totalité du débit empruntait le seul chenal sud; l'emplacement de l'ancien chenal, qui n'était plus alimenté en débit liquide par l'amont, se vidait rapidement, et la renverse jusant/flot s'y établissait près d'une heure avant celle du nouveau chenal.

On a pu constater alors une rapide évolution de l'estuaire qui se comblait dans sa partie nord, tandis que le nouveau chenal s'élargissait régulièrement entre Honfleur et la Risle. Dès le mois de décembre 1960 , la distance entre les lignes 0 était sensiblement double de sa valeur au moment du changement de chenal (fig. 11), mais le calibrage était encore insuffisant, les vitesses dans la partie centrale et amont du nouveau chenal étaient encore importantes (environ 4 à 5 nœuds), et les surcreusements du fond s'accentuaient en même temps que se développait une barre à $5 \mathrm{~km}$ en aval de Honfleur (7). De puissants dragages ont été nécessaires pour vaincre cette barre et aider l'action du flot, qui en reprenait les matériaux et les entraînait dans l'ancien chenal où ils se déposaient.

Ce n'est qu'à partir de la fin de l'année 1961, alors que les sections du nouveau chenal avaient sensiblement atteint leurs surfaces et leurs formes définitives, que les fonds de la partie comprise entre Honfleur et la Risle se sont régularisés, en même temps que la barre extérieure a diminué.

De nombreux mois ont encore été nécessaires pour réaliser le calibrage du chenal, à la fois sous l'action des courants et par l'intervention des dragues; l'érosion du banc de Saint-Sauveur dans sa partie insubmersible, protégée par la végétation, était toujours assez lente, car l'écoulement était ralenti par les faibles profondeurs de la partie nord du nouveau chenal, et sans une intervention par dragages, le calibrage aurait été très long à obtenir. En outre, le refoulement direct des produits dragués au nord de l'ancienne digue sud évitait aux matériaux le circuit par l'intermédiaire d'une barre extérieure où le flot les reprenait pour les entraîner dans l'ancien chenal.

8. Le résultat pratique le plus important de l'ouverture du nouveau chenal est évidemment l'amélioration des conditions d'accès au port de Rouen; la progression du calibrage a permis un abaissement des seuils dont le niveau était abaissé d'environ $2 \mathrm{~m}$ à la fin de l'année 1963 (fig. 12).

Sur un plan plus théorique, l'ouverture du chenal permet dès maintenant de tirer un enseigne-

(7) On a également constaté au droit de Honfleur la formation de barres très instables, constituées par des rides dont les crêtes étaient sensiblement normales à l'axe du chenal; leur amplitude atteignait $2 \mathrm{~m}$ et leur longueur d'onde variait de 20 à $30 \mathrm{~m}$; leur profl était nettement «couché sous le jusant, mais se redressait sous le flot; des sondages effectués suivant le même segment au cours de marées consécutives ont fait apparaitre une progression de ces rides vers l'aval d'une quinzaine de mètres par malées de coefficient 113. ment pouvant présenter un certain intérèt pour la réalisation des travaux de ce genre.

8.1. L'excellente concordance de l'évolution du modèle et de celle des fonds naturels, constatée Iors de l'essai historique de réglage, a également été observée dans le changement de tracé du chenal; la précision des indications du modèle réduit était cependant plus faible, en raison de la rapidité de certaines opérations dont la vitesse de réalisation en nature était nettement supérieure à la cadence des sondages effectués sur le modèle (8).

En outre, l'opération a permis de se rendre compte de la limitation des indications fournies par le modèle dans cette phase d'intense évolution qu'a été le changement de chenal; le modèle ne pouvait, en effet, prétendre à une exacte représentation de l'érosion de certains bancs insubmersibles ou de bancs constitués de matériaux de caractéristiques très variables; il ne pouvait également pas reproduire de facon absolument rigoureuse des opérations (comme le dragage de l'ancienne digue sud, ou la construction de la digue nord) qui avaient un caractère quasi instantané sur la maquette, mais dont l'exécution dura plus d'un an dans la nature.

Malgré ces limitations, les indications du modèle réduit à fond mobile se sont révélées indispensables pour déterminer l'ordre de grandeur de la durée nécessaire à l'établissement et à la stabilisation d'un nouveau chenal, et surtout pour vérifier que l'évolution avait effectivement une tendance naturelle à se faire dans le sens désiré.

8.2. L'opération réalisée dans l'estuaire de la Seine montre que l'utilisation de la méthode «progressive» comme la méthode «brutale», de même que leur combinaison, a permis de réaliser une profonde modification du tracé et des caractéristiques du chenal, malgré des conditions naturelles difficiles. Les autorités chargées du port de Rouen avaient certes une expérience de la rapide coupure des bancs de sable par l'action des forces naturelles de la marée; e'est ainsi qu'au siècle dernier, le chenal a été tracé dans un banc au droit du cap de Tancarville, et qu'en 1950 a été ouvert le nouveau chenal d'accès au port de Honfleur. Mais, dans tous les cas, et en particulier pour l'ouverture du nouveau chenal de l'estuaire, de sérieuses précautions ont été prises pour garantir au maximum le succès de l'opération en réduisant dans toute la mesure du possible la partie alćatoire de l'évolution par une préparation très poussée sur le plan des études et des travaux.

8.3. L'expérience de l'ouverture du nouveau chenal de l'estuaire de la Seine permet de formuler des recommandations qui nous paraissent essentielles pour la réalisation de tous les aménagements comportant une brutale modification d'une rivière maritime :

- Les études préalables doivent vérifier que l'écoulement a une tendance naturelle à suivre le tracé projeté, et qu'à sections mouillées équivaJentes, le débit - principalement le débit de jusant - se partage naturellement en faveur du nouveau chenal;

-.- La conception du projet et les procédés utili-

(8) Environ un sondage par année-nature d'évolution des fonds. 
sés pour sa réalisation doivent être déterminés pour que les inévitables perturbations qui se produisent pendant la phase transitoire comportant deux chenaux ouverts à l'écoulement ne risquent pas de dépasser le potentiel d'intervention par des dragages ou la construction d'ouvrages de calibrage : il faut à ce sujet porter une attention particulière à la durée de réalisation de calibrage du nouveau chenal, dont l'insuffisance peut entrainer le comblement de certaines zones en même temps que le surcreusement d'autres sections qui est souvent à l'origine de la formation de seuils très gênants pour la navigation;

- L'époque choisie pour l'exécution des travaux doit enfin permettre de les réaliser dans des conditions naturelles aussi favorables que possible. La coupure d'un banc en marée de vive-eau permet de bénéficier au mieux de l'érosion produite par de violents courants, eux-mêmes produits par une dénivellation des plans d'eau plus forte qu'en morteeau; de même l'équinoxe d'automne était, a priori, plus favorable pour l'ouverture du chenal de la Seine que l'équinoxe de printemps, car l'action du jusant, considérée comme essentielle pour l'établissement du nouveau chenal, a été renforcée par le débit fluvial qui est normalement plus important en hiver qu'en été.

D’une façon générale, on ne saurait trop insister sur la nécessité de préparer de facon aussi détaillée que possible l'exécution des travaux qui mettent en ouvre des forces de la nature, dont il convient de garder à tout instant le contrôle.

\section{Discussion}

\section{Président: M. Fischen}

M. le Président remercie M. Chapon an nom de tout l'alditoire et, rappelant que, dans les essais sur modèle réduit l'échelle des temps n'était pas la mème pour l'évolution des fonds que pour les autres phénomènes, demande à $\mathrm{M}$. Chapos comment il a pu réaliser sur modéle une évolution, en quelque sorte historique, de l'estuaire pendant une période assez longue.

M. Chapos indique que l'échelle du modèle réduit de l'estuaire de la Seine était de $8 / 100^{\text {es }}$ en plan et $1 / 100^{\circ}$ en hatuteur, et que l'échelle des temps hydrauliques reproduisait une marée de 12 h 30 en $9 \mathrm{mn}$. Cette échelle des temps hydrauliques n'avait a priori, aucune raison d'être la même que celle de l'évolution des temps de déplacement des matériaux de fond, car l'on sait bien que le débit de transport des matériaux de fond est proportionnel à la puissance $3^{\circ}$ on $4^{\circ}$ de la vitesse. Il semble qu'il n'existe pas de méthode exacte permettant de calculer a priori des temps d'évolution des fonds.

Il était très tentant de faire un essai historique dans le cas de l'estuaire de la Seine, car on disposait de plans de sondages en 1834 , en 1864 et 1865 , et ensuite pratiquement chaque année à partir de 1900; par ailleurs l'estuaire est soumis à des variations très importantes.

On a donc fait un essai historique consistant à reproduire l'évolution des fonds entre 1875 et 1953 . On pouvait done déjá connaître l'ordre de grandeur de l'échelle de l'évolution des temps par la reproduction de certains phénomènes caracéristiques, comme l'évolution de l'inflexion de Honfleur ou le colmatage des fosses de jusant. Au fur et à mesure des essais de réglage hydraulique, on s'était aperçu que la marée de coefficient 95 était, en fait, la « marée efficace » qui donnait une évolution des fonds conforme á ce qui avait été constaté dans le passé. On ne conmaissait pas encore de façon précise l'échelle des temps d'évolution des fonds, mais on voyait que si le chenal était passè d'un tracé nord à un tracé sud en l'espace de dix ans, puis était ensuite revenu à un tracé nord dix ans après,
Courbes marégraphiques

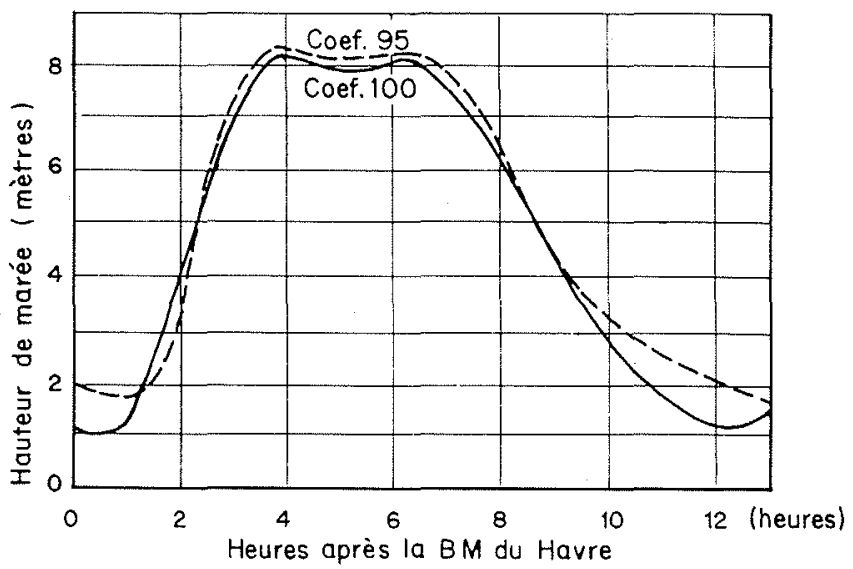

Courbes des vitesses des courants

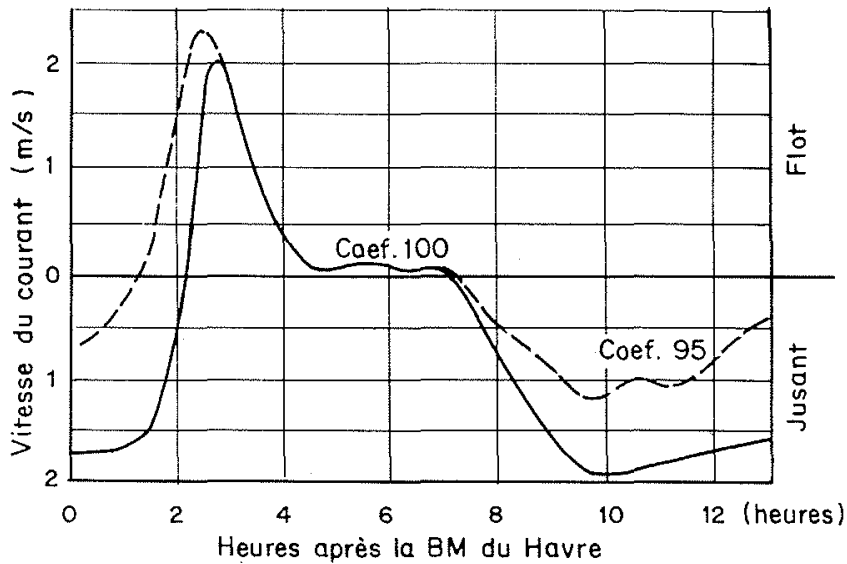

- - - Avant l'ouverture du nouveau chenal

Après

on retrouvait sur le modèle le même intervalle de temps en valeur relative.

C'est ainsi que l'on est arrivé à régler progressivement le modèle et, grâce à l'essai historique, on a pu avoir la valeur de cette échelle des temps qui était de 60 marées du modèle (pratiquement 10 heures de fonctionnement) pour un an de la nature.

Cet essai historique a, de plus, permis de verrifier que lo modèle était fidèle, sensible et juste, comme doit l'être un appareil de mesure; il était alors possible de lui faire confiance pour une longue période de l'avenir.

Au moment de la période d'ouverture, les indications données par le modèle étaient inévitablement très schématiques, car les phénomènes reproduits sur modèle réduit n'avaient qu'un rapport lointain avec ce qui se passe dans la nature: l'enlèvement d'une digue sur modèle consistait à enlever un onvrage de quelques mètres de long, alors que, dans la nature, cette digue représentait près de $300000 \mathrm{~m}^{3}$ d'enrochements. La construction de la digue nord dans l'estuaire a consisté à reposer, dans le modèle, l'ouvrage qui avait été enlevé de la digue sud, alors que dans le réel, il a fallu mettre en place plus de $500000 \mathrm{~m}^{3}$ de matériaux. Toutefois, finalement, vue sur un an, la concordance a été excellente.

M. le Président dit que cela est un résultat tout à fait remarquable.

M. Stenling indique que M. Chapon a souligné que le courant suit bien la rive; mais est-on parvenu à concentrer le courant de flot et le courant de jusant dans le mème chenal? 
M. Chapon répond que le trace des digues a précisément pour objet de réalisé le raccordement du chenal creusé par le flot contre la digue du Ratier, et celui creusé par le jusant contre la digue sud, ce racordement étant évidemment aidé par des dragarges. Il est très important, par ailleurs, de concentrer le débit de jusant dans un seul chenal, car l'action de ce courant est maximal aux cotes les plus basses de la marée.

M. Chapon souligne, à l'aide du graphique ci-contre, l'intérêt de la concentration du jusant dans un chenal unique : le flot agit, en effet, lorsque les cotes de la marée sont relativement hautes, alors que le jusant se produit dans la partie basse de la courbe des niveaux. L'aménagement de l'estuaire de la Seine a accentué cette dissymetrie d'action des niveaux de la marée en rapprochant l'instant de la pointe de flot de l'heure de la pleine mer.

L'égalité des débits liquides entrant et sortant de l'estuaire à chaque marée exige une réduction de l'intensité et de la durée du courant de flot et, au contraire, un renforcement du courant de jusant. Celte modification de la courbe des courants a pour conséquence un sérieux renforcement du ponvoir de transport solide du courant de jusant. Dans le cas particulier de l'estuaire de la Seine, ce renforcement du pouvoir de transport solide du jusant a permis de réduire la prépondérance qu'avait, dans l'estuaire libre, le débit solide de flot.

Le renforcement du debit solide de jusant doit évidemment etre conç de façon a éviter la formation d'une barre entre la fosse creusée par le flot à l'extérieur de l'estuaire et celle creusée par le jusant dans la partie amont. On a prévu a cet effet de donner à la digue nord un profil en long plongeant vers l'aval, pour raccorder de façon aussi progressive que possible la partie endiguée de l'estuaire avec la partie extérieure : le courant de jusant s'épanouit en aval de Honfleur et une partie de son débit se déverse au-dessus de la digue nord vers un banc d'équilibre qui se constitue dans le prolongement de cet ouvrage. Le désablement du jusant qui résulte de cet ópanouissement permet de raccorder de façon, a priori, assez satisfaisante la fosse de flot et la fosse de jusant.

M. le Président croit que dans ce dernier cas, on s'intéresserait encore plus au courant de jusant, car il faudrait bient évacuer les matériaux vers la mer, leur enlèvement a l'amont, que suggère M. CHAPON, pouvant etre très onéreux quand il s'agit d'une dizaine de millions de mètres cubes par an.

M. Babinet indique que M. Chapon a souligné l'importance, visible sur les plans, de la fosse qui, partant de la mer, se dirige ensuite vers le nord-est, suivant le tracé de l'ancien chenal. Cette fosse est heureusement en roie de régression. Peuton néanmoins avoir une idée de la fraction du cube, introduit par le flot, qui emprunte cette fosse?

M. Chapon répond que, dans l'état actuel des fonds de l'estuaire de la Seine et des conditions d'écoulement, on peut évaluer à $90 \%$ du débit total de flot la partie qui s'écoule vers Je nouveau chenal. La posibilité de concentrer dans le nouveau chenal la majeure partie du débit de flot de la presque totalité du débit de jusant est précisément une condition essentielle du raccordement des fosses creusées par le flot et le jusant.

Se rapportant aux courbes précéảentes, M. Chapox explique que la partie de l'estuaire comprise entre l'ancienne digue nord et la nouvelle se vide plus rapidement au moment du jusant. Le flot s'y établit très peu de temps après l'heure de la basse mer locale, alors qu'il est encore jusant dans le chenal principal. La vitesse du flot correspondant est relativement élevée mais, du fait de la faible capacité de la partie nord de l'estuaire, son remplissage est très rapide, le courant de flot $y$ dure très peu de temps et le débit correspondant est assez faible malgré des pointes de courant de l'ordre de $2,50 \mathrm{~m} / \mathrm{s}$.

M. Cotrano demande quelles sont les limites du modèle.

M. Chapon indique que le modele représentait la baie de Seine depuis le méridien de la Dives, qui est situé à environ une quinzaine de kilomètres en aval de Honfleur.

Le modèle reproduisait la Seine en vraie grandeur jusque dans la région de Quillebeuf, puis était prolongé par un serpentin qui permettait une reproduction suffisamment exacte des lignes d'eau. L'alimentation en matériaux solides était faita dans la région de la Roque (en amont du confluent de la Risle).

La schématisation des données naturelles était évidemment assez grande, mais les resultats de l'essai historique ont montré qu'elle n'aflectait pas la validité des résultats du modèle.

M. Cottand fait remarquer que des modifications dans les bancs du large sont susceptibles, par la suite, d'apporter des perturbations sérieuses dans la zone des travaux.

M. Chapox répond qu'effectivement il était nécessaire de vérifier que les conditions imposées à la limite aval du modèle ne risquaient pas d'être affectees par les modifications qui se produisaient dans l'estuaire. Une vérification theo. rique, qui ne prétendait d'ailleurs donner aucun ordre de grandeur, a été effectuée en intégrant de façon numérique les équations de propagation de la marée. Sur le plan mathématique, le problème revient à résoudre un problème de Neuman intérieur.

On a ensuite vérifié par un calcul précis, puis par des essais sur le modèle réduit de la Manche (échelle $1 / 50000^{\circ}$ ) des Laboratoires de Chatou, que d'importantes modifications, pouvant aller jusqu'à un colmatage presque total de l'estuaire, n'apportaient aucune modification décelable à quelques kilomètres en aval du méridien du cap de la Hève. Des vérifications récemment effectuées en nature ont confirmé les résultats précédents.

M. Stenting se demande si la rupture d'équilibre vers l'amont n'est pas beaucoup plus importante que celle vers l'aval. Jusqu'à l'amont, n'y a-t-il pas des perturbations qui sont des améliorations, mais qui s'écartent de l'état actuel?

M. Charon dit que les modifications des lignes d'eau constatées dans la partic amont de la rivière, dues à l'exécution des travaux de l'estuaire, sont insignifiantes. L'abaissement du lieu géométrique des basses mers auquel on pouvait s'attendre a été relativement faible. Il semble que l'aménagement de l'estuaire ait entrainé une légère augmentation du marnage dans la partic aval, mais n'affecte pas la région située au-dela d'une quarantaine de kilomètres en amont de l'embouchure.

Tontefois, J'évolution des fonds de l'estuaire a été progressive ef des vérifications sont actuellement en cours pour déterminer les modifications apportées par l'aménagement aux courbes des marées de la rivière (en particulier en ce qui concerne les harmoniçues).

Il semble, par contre, que le niveau de la basse mer dans la rivière soit plus sensible à l'approfondissement local du lit par le dragage des fonds inaffouilables.

M. le président demande si cette réaction a été calculée ou mesurée en tenant compte des dragages d'entretien que l'on a effectués et de ceux que l'on sera conduit à faire dans cette partie.

M. Chapon indique que les dragages d'entretien dans l'estuaire de la Seine consistent à corriger les inégalités du chenal, et se ramènent, en fait, à un déplacement des matériaux à l'intérieur de l'estuaire sur des distances relativement faibles. Il ne semble pas que l'enlèvement de buttes isolées de faibles dimensions, qui sont évidemment fort gênantes pour la navigation, puisse modifier les conditions d'écoulement et, par conséquent, affecter les lignes d'eau.

Sur une remarque de M. le Président, M. Chapos dit que les dragages d'entretien faits sur le modèle représentaient théoriquement des quantités comprises entre 4 et 6 millions de metres cubes par an. Il ne faut toutefois pas accorder une signification trop précise a l'importance de ces quantités, car les conditions de dragages du modèle ne pouvaient prétendre représenter exactement l'action des dragues aspiratrices en marche, et il était admis que les quantités qui seraient draguées dans le réel pourraient être supérieures aux précédentes, précisément pour tenir compte de l'effet d'amélioration relativement lente apporté par les dragues aspiratrices, beacoup moins favorables que les dragages a caractère plus massif effectués sur le modèle.

Actuellement, les dragages réalisés représentent 7 à 8 millions de métres cubes par an, mais certains d'entre eux consistent a déraser les parties inaffoullables du lit ou a achever la démolition d'anciens ouvrages.

Le modèle a montré qu'après la stabilisation du chenal, la majeure partie des dragages devrait être effectuée sur la barre d'embouchure, où les matériaux seraient déposés par le courant de jusant dont la vitesse diminuera ver's l'aval.

L’aménagement a été étudié de facon à épanouir suffisamment le débit solide du jusant et à répartir en conséquence les dépots de matériaux, les dragages étant, au contraire, effectués en concentrant l'action des engins sur une zone de faible superficie.

M. le Président remercie à nouveau M. Chapon pour toutes ses explications. 


\section{Abstract \\ The creation of a new access channel to Rouen harbour in the Seine Estuary \\ by J.-P. Chapon *}

1. The improvement of navigation conditions in a tidal river frequently equires major changes to be made to its channel position and characteristics, and the less the natural conditions lend themselves to the maintaining of a deep channel, the more radical the changes required. Generally speaking, these can be achieved either by the progressive construction of training works or by a sudden cutting made to rapidly connect up two bodies of water separated by banks or dyles.

Both methods were resorted to in opening up the new Rouen harbour shipping channel, by first cutting openings in a dyke and a sand bank and then following up with progressive construction work.

2. The most delicate part of the operation was to change the channel between Honfleur and the Risle confluence, which had to be done without interrupting navigation on the river. This was achieved by excavating a thirty-million cubic metre capacity basin out of an emergent sand bank and by connecting this basin to the estuary, first by the sudden piercing of an opening in its southern end, and then by gradually opening up its northern end as well. The embankment marking the future channel ran along the southern edge of the basin.

3. The diversion of increasing proportions of the tidal flow into the new channel was made easier not only by the progressive closure of the old chamel, but also because the new channel layout backed up against a concave embankment, and because of the increased flow cross-section and lower flow resistance resulting from dredging operations carried on at the time.

4. Considerable unwelcome deep scouring is likely to occur where flows are concentrated in narrow cross-sections; as the banks this forms are a shipping hazard, such conditions should only be allowed to remain for the minimum possible length of time.

The "sudden" method scores to some extent in this respect, and was already used successfully during the last century to open the Tancarville bend in the tidal part of the Seine. A more recent example was the opening of a new channel at Honfleur. It should not be used, however, unless it has been confirmed - for instance by tests on a loose-bed model - that the flow will have a natural tendency to follow the new channel, and that the available dredding facilities provide an adequate means of correcting the channel irregularities that are bound to occur until it has finally settled down.

5. Generally speaking, any major modification of the alignment and dimensions of a river with a wide tidal range is necessarily a difficult undertaking. Its success depends on long painstaking study, a plan of operations prepared down to the last detail, and every possible use of natural forces, over which full contro! must be maintained at all times.

\footnotetext{
* Ingénieur des Ponts et Chaussées à la Direction du Port de Rouen.
} 